\title{
Queuine, a bacterial derived hypermodified nucleobase, shows protection in in vitro models of neurodegeneration
}

Patricia Richard ${ }^{1 *}$, Xavier Manière ${ }^{2 *}$, Lucie Kozlowski², Hélène Guillorit ${ }^{2,3}$, Patrice Garnier², Antoine Danchin ${ }^{4}$, Nicole C. McKnight ${ }^{1 * *}$

${ }^{1}$ Stellate Therapeutics Inc., 101 Avenue of the Americas, JLABS @ NYC, New York, NY 10013

${ }^{2}$ Stellate Therapeutics SAS, 47 rue de Montmorency, 75003, Paris, France

${ }^{3}$ Institut de Génomique Fonctionnelle, 141 rue de la Cardonille, 34094, Montpellier, France

${ }^{4}$ Kodikos Labs, Institut Cochin, 24 rue du Faubourg Saint-Jacques, 75014, Paris, France

** corresponding author

Email: ncm@stellate-tx.com

* These authors contributed equally to this work 


\begin{abstract}
Growing evidence suggests that human gut bacteria, comprising the microbiome that communicates with the brain through the so-called 'gut-brain-axis', are linked to neurodegenerative disorders. Imbalances in the microbiome of Parkinson's disease (PD) and Alzheimer's disease (AD) patients have been detected in several studies. Queuine is a hypermodified nucleobase enriched in the brain and exclusively produced by bacteria and salvaged by humans through their gut epithelium. Queuine replaces guanine at the wobble position of tRNAs with GUN anticodons and promotes efficient cytoplasmic and mitochondrial mRNA translation.

To elucidate whether queuine could facilitate protein folding and prevent aggregation and mitochondrial defects, hallmarks of neurodegenerative disorders, we tested the effect of chemically synthesized queuine, STL-101, in several in vitro models of neurodegeneration. Treatment with STL-101 led to increased neuronal survival as well as a significant decrease in hyperphosphorylated alpha-synuclein, a marker of alpha-synuclein aggregation in a PD model and a decrease in tau hyperphosphorylation in an AD model. Our work has identified a new role for queuine in neuroprotection uncovering a therapeutic potential for STL-101 in neurological disorders.
\end{abstract}




\section{Introduction}

Parkinson's and Alzheimer's diseases (PD and AD) are the most common neurodegenerative diseases, affecting seven and 44 million people worldwide respectively. PD is characterized by classical motor difficulties and non-motor manifestations that can occur years before diagnosis. Gastrointestinal symptoms such as constipation are among the main non-motor symptoms in PD that often occur a decade or more before onset [1]. Recently several studies showed that hallmarks of neurodegeneration such as Lewy bodies are found in the enteric nervous system (ENS) [2] and Parkinson's disease patients endure gut dysbiosis [3,4]. Alzheimer's disease is the leading cause of dementia and is characterized by several clinical symptoms that include a progressive decline in memory, thinking, speech and learning capacities. Alzheimer's disease patients also show a change in their microbiota compared to healthy individuals [5-7]. Gut microbiota transfer in mouse models of PD and AD highlighted a functional link between bacteria composition and neurodegeneration [8,9]. Altogether, a widespread understanding of these data is that communication between the gut and the brain, namely the gut-brain axis [10], is linked to disease.

Bacteria that have co-evolved with Eukaryotes are a source of many unique molecules that connect the gut with several organs including the brain, impacting human health and brain function. Queuine is a pyrrolopyrimidine containing analog of guanine that is exclusively synthesized by bacteria and found in most eukaryotes, including humans who acquire queuine from their own gut microbiota and a diet containing this bacterial derived molecule [11]. Queuine produced by the gut microbiota is salvaged by humans through their gut epithelium and is distributed to a wide range of tissues including the brain where it is enriched [12]. Queuine is incorporated into the wobble position (nucleoside 34) of cytoplasmic and mitochondrial transfer RNAs (tRNAs) containing a $\mathrm{G}_{34} \mathrm{U}_{35} \mathrm{~N}_{36}$ ( $\mathrm{N}=$ any nucleotide) anticodon sequence $[13,14]$. In turn, its corresponding nucleoside queuosine is found in four specific tRNAs acceptors for the amino acids asparagine, aspartic acid, histidine and tyrosine $[11,15,16]$. Queuine modification of tRNA (QtRNA) is an irreversible event [17] that occurs through a unique base-for-base exchange reaction (guanine replacement by queuine) by the eukaryotic heterodimeric tRNA guanine transglycosylase (TGT) enzyme composed of the catalytic QTRT1 and accessory QTRT2 subunits [18]. Like most 
post-transcriptional modifications in the anticodon loop of tRNAs, especially position 34, queuosine is critical for the translation process [19]. Queuine incorporation into tRNA regulates translational speed and maintains accuracy [14,20-22]. Deregulation of translation upon queuine depletion in both human and mice cells results in the formation of unfolded proteins that trigger endoplasmic reticulum (ER) stress and activation of the unfolded protein response (UPR) $[14,21]$. Mitoribosome profiling in QTRT2 KO cells recently showed that queuine at position $34(\mathrm{Q} 34)$ controls elongation speed in mitochondria in a similar way than in the cytoplasm [14]. The 13 identified proteins translated in mitochondria are all components of the oxidative phosphorylation machinery [23] and a defect in mitochondrial protein homeostasis would dramatically compromise cellular metabolism. Mitochondrial dysfunction is indeed linked to several neurological disorders including PD and AD [24].

Another interesting link between queuine and PD is its requirement in maintaining a normal level of tetrahydrobiopterin (BH4), an essential co-factor for aromatic amino acid hydroxylases such phenylalanine hydroxylase (PAH) and tyrosine hydroxylase (TH). PAH synthesizes tyrosine from phenylalanine, which is then hydroxylated by TH to catalyze the formation of levodopa (L-DOPA), the precursor of dopamine [25]. PD is characterized by the death of dopaminergic neurons in the substantia nigra (SN), consequently leading to a dopamine deficiency that contributes to movement problems. While mice deprived of queuine appear asymptomatic, germ-free mice fed with a diet deficient in both tyrosine and queuine develop neurological abnormalities and die before 18 days [26]. Additionally, TGT-deficient mice have decreased levels of $\mathrm{BH} 4$ and elevated levels of its oxidized form BH2 (dihydrobiopterin), compromising tyrosine production, which indicates a crucial role for queuine in tyrosine metabolism [27]. BH4 is also a central co-factor for tryptophan hydroxylase (TPH) that leads to the production of serotonin, a neurotransmitter also deficient in PD patients [28]. While queuosine appears to block BH4 oxidation, queuine has also shown to protect HeLa cells against oxidative stress, another known contributor to neurological cell degeneration in PD [29,30]. These findings imply that queuine plays an important role in dopamine synthesis through $\mathrm{BH} 4$ metabolism and prevention of oxidative stress. 
Because queuine is enriched in the brain and promotes optimal cytoplasmic and mitochondrial mRNA translation necessary to prevent protein aggregation, a hallmark of several neurological disorders, we investigated the potential protective effect of synthesized queuine in several models of neurodegeneration.

\section{Results}

Synthesized queuine decreases alpha-synuclein hyperphosphorylation and is neuroprotective in a neurotoxin-based model of PD.

Because queuine promotes optimal translation and gut microbiome changes have been linked to PD in several studies [4], we first investigated the effect of synthesized queuine (hereafter referred as STL-101)[31] in an in vitro model of synucleinopathy. Exposure of primary neuronal cells to recombinant human pre-formed alpha-synuclein ( $\alpha$-syn) fibrils (huPFFs) leads to the aggregation of endogenous $\alpha$-syn into Lewy Bodies, a hallmark of PD [32]. We cultured wildtype (WT) primary mouse cortical neurons with or without STL-101 followed by huPFF exposure for three weeks and monitored $\alpha$-syn aggregation by immunoflourescence (IF) staining of $\alpha$-syn phosphorylated at serine 129 (Ser129), a surrogate marker of de novo $\alpha$-syn aggregation [33] (Fig 1). The Syn-211 antibody specifically detects human $\alpha$-syn while the EP1536Y antibody specifically detects Ser129 phosphorylated form of $\alpha$-syn ( $\alpha$-syn pSer129) of both human and rodent. After three weeks of huPFF exposure, EP1536Y staining detected Lewy neurites and $\alpha$-syn aggregates in the neuronal cell bodies of WT neurons exposed to huPFFs while only background signal was observed in control conditions (Fig 1A). Strikingly, STL-101 pre-treatment of huPFF exposed neurons led to significantly decreased $\alpha$-syn pSer129, indicating a strong decrease in $\alpha$-syn aggregation (Fig 1B). Quantification of EP1536Y antibody signal showed a $\sim 40 \%$ decrease of $\alpha$-syn pSer129 in cells pre-treated with STL-101 at $100 \mathrm{nM}$ and up to $50 \%$ in cells treated with $10 \mu \mathrm{M}$ of STL-101 compared to control cells not treated with STL-101 (Fig 1C).

In order to test the robustness of the effect of STL-101, we tested it in a different model of PD using the neurotoxin MPP+ (1-methyl-4-phenylpyridinium), which induces degeneration of 
dopaminergic (DA) neurons. MPP+ inhibits mitochondrial complex I leading to the production of reactive oxygen species (ROS) by mitochondria and subsequent death of DA neurons [34]. Rat DA neurons were cultured for six days in vitro (DIV) and treated with STL-101 at several concentrations (from $0.003 \mu \mathrm{M}$ to $30 \mu \mathrm{M}$ ) one day before the addition of MPP+. Two days later, survival of DA neurons was assessed by counting TH+ neurons (Fig 1D). While MPP+ treatment resulted in $50 \%$ of DA neuron death, STL-101 treatment at $1 \mu \mathrm{M}$ and above significantly rescued DA neuron death leading to $65-85 \%$ survival of $\mathrm{TH}+$ neurons.

These results show that STL-101 is protective in two distinct systems widely used to study PD, bringing to light its therapeutic potential in humans suffering from proteinopathy and neurodegeneration.

\section{STL-101 reduces tau hyperphosphorylation and cell death in in vitro models of AD.}

Alzheimer's disease is characterized by extracellular amyloid beta $(A \beta)$ ( $\sim 0$ amino acid long peptide cleaved from the amyloid- $\beta$ precursor protein (APP)) accumulation forming amyloid plaques, hyperphosphorylation and aggregation of tau forming intracellular neurofibrillary tangles (NFTs), and neuroinflammation. Increased levels of $A \beta\left(A \beta_{1-40}\right.$ and $A \beta_{1-42}$ being the most abundant forms) can be found in the brain of $A D$ patients and animal models and it is the $A \beta$ oligomers that are believed to be the cause or at least contribute to the toxicity in AD pathogenesis [35]. AD is also associated with microglia activation, referred to as microgliosis, which is observed early in disease progression. First, in order to evaluate STL-101 capacity to reduce A $\beta$ toxicity, we used an acute $A \beta_{1-42}$ oligomer injury model that reproduces essential neuropathological features of $A D$ and a neuroinflammatory response [36]. A $\beta$ peptides are produced as soluble monomers and undergo oligomerization with amyloid fibril formation via a nucleation-dependent polymerization process [37]. STL-101 was added to primary cultures of rat cortical neurons ten days after seeding and one day before $A \beta_{1-42}$ injury at $20 \mu \mathrm{M}$. 24 hours after $A \beta_{1-42}$ injury, IF staining of MAP-2 (Microtubule Associated Protein 2) and hyperphosphorylated tau (p-tau) was performed to evaluate survival of cortical neurons, neurite network and $p$-tau (Fig $2 A$ ). While $A \beta_{1 \text { - }}$ 
42 injury led to a significant increase in p-tau compared to control, treatment with STL-101 strongly reduced p-tau in a dose dependent manner (Fig 2B). STL-101 also protected the neurite network which is normally altered by $A \beta_{1-42}$ injury (Fig 2C). Importantly, pre-treatment of STL-101 at a concentration of 30nM and above showed neuroprotection, increasing cell survival from approximately $70 \%$ to $80-85 \%$ at concentrations of $0.3 \mu \mathrm{M}$ and $1 \mu \mathrm{M}$.

In addition, we tested the effect of STL-101 in both rat primary neuronal culture and microglia treated with $A \beta_{1-42}$ for $72 \mathrm{~h}$ at $5 \mu \mathrm{M}$ (S1 Fig). Similar to the effect after acute injury, chronic injury led to a significant increase in the neurite network at concentrations of STL-101 of $1 \mu \mathrm{M}$ and above (S1A Fig) and a decrease in p-tau at concentrations of STL-101 of $0.3 \mu \mathrm{M}$ and above (S1B Fig). We observed an associated neuroprotective effect of STL-101, however it was not statistically significant (S1C Fig). Of interest, TNF- $\alpha$ release in the culture medium induced by $A \beta_{1-42}$ injury was significantly lower in

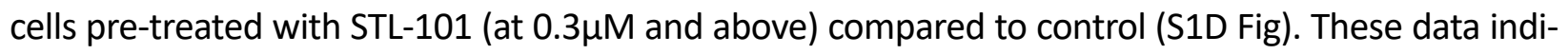
cate that STL-101 not only exhibits neuroprotective properties but also shows an anti-inflammatory effect in a chronic model of AD.

\section{Queuine level is not age-dependent but higher in women}

Neurodegenerative disorders are very often associated with aging. To determine any agerelated or sex differences in the level of queuine, we measured queuine in the plasma of 80 neurologically healthy men and 80 neurologically healthy women from 50 to 90 years old. Measurement of queuine levels by LC-MS/MS showed no significant decline of queuine in plasma with increasing age (Fig 3A). This data is in perfect keeping with healthy individuals maintaining a protective queuine level while a decrease in queuine availability is likely occurring in patients experiencing dysbiosis. Unexpectedly, we found significantly higher level of queuine in the plasma of women compared to men (Fig 3B). Interestingly, analysis of the queuine levels in the plasma of 45 weeks old mice ( 1 year old) also showed a higher level in female ( $>3$ times more) than male (S2 Fig). 


\section{Discussion}

Here we have shown remarkable protective properties of queuine, a molecule exclusively produced by bacteria, in several in vitro models of proteinopathies and neurodegeneration. We first showed that pre-treatment of huPFFs-exposed neurons with chemically synthesized queuine (named STL-101 in this work), decreased $\alpha$-syn pSer129 by approximately $50 \%$. This indicates a strong reduction or prevention of $\alpha$-syn aggregation normally triggered by exogenous huPFFs. We also showed a neuroprotective role of STL-101 in the MPP+ neurotoxin model of PD and in both acute and chronic models of AD. STL-101 pre-treatment rescued about $15-35 \%$ of MPP+-exposed DA neurons from death. The neuroprotective effect identified in the acute AD model was associated with a decrease in phosphorylated tau and presumably the formation of NFTs, hallmarks of $A D$. Of importance, the chronic $A \beta_{1-42}$ injury model revealed a lower production of pro-inflammatory cytokine in STL-101-treated cells. Together these data indicate that treatment with STL-101 offers several beneficial effects in various neurodegeneration models for which protein aggregates are a common feature. While we tested STL-101 as a preventive treatment, it will next be important to test whether STL-101 offers similar benefits when administrated post injury.

Interestingly, we showed that the level of queuine does not vary with age in healthy individuals and that the level is higher in women than men. It is well established that neurodegenerative diseases often show sex bias [38], for instance PD and amyotrophic lateral sclerosis (ALS) are more prevalent in men than in women while it is the opposite in $A D$ and multiple sclerosis. It will be interesting to investigate whether these sex biases could be explained by the difference in queuine distribution and levels. This also highlights the importance of stratifying patients in order to select the subpopulation that would most likely benefit from specific therapy such as STL-101 treatment. Comparing queuine levels in biofluids and tissues of neurologically healthy individuals with patients affected by neurodegenerative disorders such as $A D$ and PD will be of particular importance to substantiate our hypothesis that neurodegeneration is linked to queuine deficiency possibly triggered by dysbiosis. Importantly, seeing similar sex difference in queuine concentration in the plasma of mice and humans indicate that mouse models will be suitable to further perform pharmacodynamics and pharmacokinetics studies. 
Many disorders associated with defects in tRNA modification have been described and include metabolic diseases, respiratory defects, myopathies as well as several mitochondrial disorders [39]. On average, a tRNA contains 13 post-transcriptional modifications and a total of about 100 modifications have been identified in tRNAs, often in or adjacent to the wobble position that is crucial for mRNA decoding [40]. The human brain is highly sensitive to defects in tRNA modification and several neurological disabilities are linked to mutations in genes responsible for tRNA post-transcriptional modifications [41]. It is then not surprising that a well-conserved and complex modification such as queuosine replacing guanosine gives advantageous benefits and protection in the brain that needs to quickly adapt to a fast-changing environment and high frequency of oxidative stress.

Another way for Q-tRNA to regulate mRNA translation is through its protection from cleavage into tRNA fragments that can interfere with translation. Indeed tRNAs lacking queuine modification are especially vulnerable to stress-induced cleavage by the ribonuclease angiogenin (ANG) in the anticodon loop [42,43]. In fact, tRNAs undergo cleavage by several endonucleases leading to the production of a multitude of stable tRNA fragments (tRFs) of various sequences and sizes [44]. Among those tRFs, 5' tRNA halves and 3' tRNA halves (also named tRNA-derived stress-induced RNA or tiRNA), both products of ANG cleavage, are highly abundant under stress conditions $[42,45]$ and have been found to inhibit protein synthesis $[46,47]$. Interestingly, tRNA halves have also been detected in the serum and CSF (cerebrospinal fluid) of PD patients and proposed to be used as biomarkers [48]. This role of queuine in tRNA cleavage inhibition and translation regulation can be expanded to its role in stimulating other tRNA modifications such as methylation of C38 in tRNA Asp as shown in S. pombe [49]. Similar to Q34, C38 has been shown to inhibit stress-induced tRNA cleavage by RNA endonucleases $[43,50]$. Not only can queuine regulate stability of a pool of specific tRNAs but it can also contribute to protein synthesis inhibition through tRFs synthesis under stress condition $[46,47]$. The protective role of queuine against tRNA cleavage is however in contrast with studies showing a neuroprotective role of ANG [5153] and the fact that mutations in ANG have been linked to PD and ALS [54]. Nevertheless, ANG overexpression in the substantia nigra of mice model of PD slightly exacerbates dopamine neuronal death compared to controls [55]. 
While Q-tRNAs function in cytoplasmic and mitochondrial mRNA translation speed and fidelity, the mechanism leading to the protective effect on neurons is uncertain. TGT KO mice are viable and largely asymptomatic but do show symptoms similar to those associated with phenylketonuria (PKU) in that they are deficient in tyrosine production from phenylalanine and $\mathrm{BH} 4$ levels are significantly decreased in the plasma, while the $\mathrm{BH} 4$ oxidation product dihydrobiopterin ( $\mathrm{BH} 2)$ is elevated [27]. PKU results from a mutation in phenylalanine hydroxylase leading to phenylalanine buildup and associated neurological problems. While reduced $\mathrm{BH} 4$ levels have been linked to PD $[25,56]$, the exact mechanism through which queuine/queuosine impacts $\mathrm{BH} 4$ remains to be elucidated.

Aberrations in mitochondrial function can contribute to the molecular pathogenesis of both PD [57] and AD [58]. Mitochondria control translation of 13 subunits of the electron transport chain essential for oxidative phosphorylation [59] and the biogenesis of iron-sulfur cluster (ISC) essential for a broad range of cellular functions [60]. The mitochondrial unfolded protein response (UPR ${ }^{\mathrm{mt}}$ ) has been found to be activated in cortexes of $A D$ patients and enhancement of mitochondrial function and proteostasis shows a reduction in $A \beta$ aggregation and toxicity [61]. To potentially ensure proper translation of $\alpha$-syn, tau or even APP, it is very likely that queuosine assures quality control of mitochondrial translation and homeostasis. A recent study found that queuine depletion promotes mitochondrial dysfunction resulting in an increased rate of proton leak and a decrease in ATP production in HeLa cells [62].

Queuine deficiency has also been linked to an increased progression of several human cancers [63]. Interestingly, an analog of queuine has already shown remarkable effect in an autoimmune disease mouse model of multiple sclerosis (EAE) by reversing disease hallmarks [64]. These reports and our work presented here demonstrate the therapeutic potential of chemically synthesized queuine (STL-101) possibly as a director of both general and specific mRNA translation quality control and may play a crucial role in optimal brain function and in protection against proteinopathies in particular. To confirm this hypothesis and further understand the molecular mechanisms behind such function, we will continue experiments with STL-101 including in vivo mouse models of neurodegeneration. 


\section{Material and Methods}

\section{STL-101 synthesis}

STL-101 has been synthesized by Synthenova SAS following the protocol established by Brooks et al. [31].

\section{Primary culture of cortical neurons and huPFFs challenge}

Timed pregnant female mice were received from Charles River Laboratories 2 days before initiation of the primary culture. Cortices were harvested from the E18 embryos of WT mice and dissociated enzymatically and mechanically to yield a homogenous cell suspension. 20,000 cells were plated per well in poly-D-Lysine-coated 96-well plates in a neuronal medium containing $0.5 \%$ Penicillin/Streptomycin and 0.5mM L-glutamine. The cultures were incubated at $37{ }^{\circ} \mathrm{C} / 5 \%$ CO2. At DIV 3 they were exposed to STL-101 at 3 concentrations $(10 \mu \mathrm{M}, 1 \mu \mathrm{M}, 0.1 \mu \mathrm{M})$ or none. STL-101 treatment was then renewed every 3 days (by replacing 1/3 of the volume of medium with fresh medium supplemented with 1 X concentration of STL-101). At DIV 7, half of the culture were exposed to huPFFs (at 1 concentration, i.e. equivalent to $10 \mathrm{nM} \alpha$-syn monomer). At 30 DIV, the neuronal cultures were fixed with PFA, and the effects of the treatments were evaluated by performing two double immunostainings: (i) D37A6/Syn1 to detect total rodent $\alpha$-syn/human plus rodent non aggregated $\alpha$-syn. (ii) EP1536Y/Syn211 to detect rodent and human $\alpha$-syn phosphorylated at S129/total human $\alpha$-syn. The induction of $\alpha$-syn phosphorylation was quantified by measuring the accumulation of phospho- $\alpha$-synuclein (length of the phospho- $\alpha$-synuclein-positive neurite network, number of phospho- $\alpha$-synuclein positive cell bodies). The experimental conditions were performed in quadruplicate (for STL-101-treated conditions +/- huPFFs) and triplicate (for untreated +/- huPFFs) wells. An array of nine individual microscopic fields were acquired in each single well, and 3 channels were recorded for each field (green fluorescence excited@488 nm, red fluorescence excited @594nm, and phase contrast).

\section{Culture of mesencephalic dopaminergic neurons, MPP+ treatment and immunostaining}

Cultures were prepared from the ventral mesencephalon of 15.5 days Wistar rat embryos (Janvier Breeding Center, Le Genest St Isle, France). Dissociated cells in suspension obtained by 
mechanical trituration of midbrain tissue pieces were seeded at a density of $1.2-1.510^{5} \mathrm{cells} / \mathrm{cm}^{2}$ onto tissue culture supports pre-coated with $1 \mathrm{mg} / \mathrm{mL}$ polyethylenimine diluted in borate buffer pH 8.3 as described in Michel et al., 1997. cultures Cere maintained in Neurobasal medium supplemented with B27, $2 \mathrm{mM} \mathrm{L-glutamine,} 100 \mathrm{mg} / \mathrm{ml}$ streptomycin, and $100 \mathrm{U} / \mathrm{ml}$ penicillin as well as $\mathrm{K}^{+}(30 \mathrm{mM})$ and $\mathrm{MK}-801(5 \mu \mathrm{M})$ to avoid excitotoxicity. Note that tyrosine hydroxylase $\left(\mathrm{TH}^{+}\right)$ neurons represent approximately $1-2 \%$ of the total number of neuronal cells present in these cultures.

STL-101 was added at DIV6 and 1 day before MPP+ addition at 10 $\mu$ M. 48 hours following MPP ${ }^{+}$ treatment, cultures were fixed for 12 min using $4 \%$ formaldehyde, then washed twice with PBS before an incubation step at $4^{\circ} \mathrm{C}$ for $24-48 \mathrm{~h}$ with an anti-TH antibody produced in mouse diluted 1/5000 (anticorps-enligne, \#ABIN617906) to assess the number of DA neurons; Detection of antiTH antibody was performed with a secondary anti-mouse CF-488 antibody (1/500, Ozyme, \#BTM20208). $\mathrm{TH}^{+}$neurons counting is performed at $\times 200$ using a $\times 20$ objective matched with a $\times 10$ ocular. The number of $\mathrm{TH}^{+}$neurons in each culture well is estimated after counting 6 visual fields distributed along the $x$ and $y$ axes.

\section{Culture of cortical neurons for $A \beta_{1-42}$ acute exposure}

Rat cortical neurons were cultured as described by Callizot et al., 2013 [36]. Briefly pregnant female rat (Wistar) of 15 days of gestation was deeply anesthetized and then sacrificed. Fetuses were collected and immediately placed in ice-cold L15 Leibovitz medium with a 2\% penicillin $(10,000 \mathrm{U} / \mathrm{mL})$ and streptomycin $(10 \mathrm{mg} / \mathrm{ml})$ solution (PS) and $1 \%$ bovine serum albumin (BSA). Cortex were treated for $20 \mathrm{~min}$ at $37^{\circ} \mathrm{C}$ with a trypsin-EDTA solution at a final concentration of $0.05 \%$ trypsin and $0.02 \%$ EDTA. The dissociation was stopped by addition of Dulbecco's modified Eagle's medium (DMEM) with 4.5g/L of glucose, containing DNAse I grade II (final concentration $0.5 \mathrm{mg} / \mathrm{mL}$ ) and $10 \%$ fetal calf serum (FCS). Cells were mechanically dissociated by three forced passages through the tip of a $10 \mathrm{~mL}$ pipette. Cells were then centrifuged at $515 \mathrm{xg}$ for $10 \mathrm{~min}$ at $4^{\circ} \mathrm{C}$. The supernatant was discarded, and the pellet was resuspended in a defined culture medium consisting of Neurobasal medium with a $2 \%$ solution of B27 supplement, $2 \mathrm{mmol} / \mathrm{L}$ of L-glutamine, 
$2 \%$ of PS solution, and $50 \mathrm{ng} / \mathrm{mL}$ of brain-derived neurotrophic factor (BDNF). Viable cells were counted in a Neubauer cytometer, using the trypan blue exclusion test. The cells were seeded at a density of 25,000 per well in 96-well plates precoated with poly-L-lysine and were cultured at $37^{\circ} \mathrm{C}$ in an air (95\%) - $\mathrm{CO}_{2}(5 \%)$ incubator. For 96 wells plates, only 60 wells were used. The wells of first and last lines and columns were not used (to avoid the edge effect) and were filled with sterile water. The medium was changed every 2 days.

\section{STL-101 treatment and human $A \beta_{1-42}$ exposure}

On day 10 of culture, STL-101 was dissolved in the culture medium and pre-incubated with primary cortical neurons for 24 hours, before $A \beta_{1-42}$ exposure. STL-101 was tested at 0.01, 0.03, 0.1, $0.3,1$ and $3 \mu \mathrm{M}$. Then, on day 11 of culture, the cortical neurons were injured with $A \beta$ solutions. The $A \beta_{1-42}$ preparation was done following the procedure described by Callizot et al., 2013. The batch number of the $A \beta_{1-42}$ was used in this study contains $10 \%$ of oligomers (quantification by WB). Briefly, $A \beta_{1-42}$ peptide was dissolved in the defined culture medium mentioned above, at an initial concentration of $40 \mu \mathrm{mol} / \mathrm{L}$. This solution was gently agitated for 3 days at $37^{\circ} \mathrm{C}$ in the dark and immediately used after being properly diluted in culture medium to the concentration used $(20 \mu \mathrm{M}, 2 \mu \mathrm{M}$ oligomers evaluated by $\mathrm{WB}) . A \beta_{1-42}$ preparation was added to a final concentration of $20 \mu \mathrm{M}(2 \mu \mathrm{M}$ oligomers, $\mathrm{A} \beta O)$ diluted in control medium in presence of the compound, for 24 hours.

\section{Immunostaining after $A \beta_{1-42}$ exposure}

24 hours after $A \beta$ application, the cell culture supernatant was removed and discarded, and the cortical neurons were fixed by a cold solution of ethanol (95\%) and acetic acid (5\%) for 5 min at $20^{\circ} \mathrm{C}$. The cells were washed twice in PBS, permeabilized, and non-specific sites were blocked with a solution of PBS containing $0.1 \%$ of saponin and $1 \%$ FCS for $15 \mathrm{~min}$ at room temperature. After permeabilization with $0.1 \%$ of saponin, cells were incubated for 2 hours with: (i) chicken polyclonal antibody anti microtubule-associated-protein 2 (MAP-2) (Abcam, \#Ab5392) at dilution of $1 / 1000$ in PBS containing $1 \%$ fetal calf serum and $0.1 \%$ of saponin (this antibody stains specifically cell bodies and neurites, allowing study of neuronal cell death and neurite network). (ii) 
mouse monoclonal anti-hyperphosphorylated tau (Thr212, Ser214; AT100) (Fisher Scientific, $\# 11818711$ ) at the dilution of $1 / 100$ in PBS containing $1 \%$ fetal calf serum and $0.1 \%$ of saponin. These antibodies were revealed with secondary antibodies goat anti-mouse IgG (Sigma, \#SAB4600042) and goat anti-chicken IgG (Sigma, \# SAB4600079) coupled with an Alexa Fluor at the dilution $1 / 400$ in PBS containing $1 \%$ FCS, $0.1 \%$ saponin (1 hour at RT).

For each condition, 30 pictures/well (representative of the whole well area) were automatically taken using ImageXpress (Molecular Devices) at 20x magnification. All images were generated by ImageXpress ${ }^{\circledR}$ using the same acquisition parameters. From images acquisition, analyses were directly and automatically performed by Custom Module Editor ${ }^{\circledR}$ (Molecular Devices). The following analysis were performed with Custom Module Editor ${ }^{\circledR}$ (Molecular Devices):

- Total neuron survival (number of MAP-2 positive neuron)

- Total neurite network (length of MAP-2 positive neurite in $\mu \mathrm{m}$ )

- Hyperphosphorylation of Tau in neuron (AT100, overlapping Tau/MAP-2 expressed in area, $\mu \mathrm{m}^{2}$ )

\section{Cell preparation, $A \beta_{1-42}$ exposure, immunostaining and TNF- $\alpha$ quantification in the chronic model of AD}

Cortical neurons were prepared as described above for the $A \beta_{1-42}$ acute exposure. STL-101 treatment, $A \beta_{1-42}$ exposure and immunostaining were also performed as described for the acute model with the following modifications:

- $A \beta_{1-42}$ solution was diluted in culture medium at $5 \mu \mathrm{M}(0.5 \mu \mathrm{M}$ oligomers, $A \beta O)$ in the presence of STL-101 for 72 hours

- $\quad$ STL-101 was tested at 0.03, 0.1, 0.3, 1 and $3 \mu \mathrm{M}$

- $\quad$ AT-100 was used at $1 / 400$

In order to assess the activation of microglia in the cell culture, the level of TNF- $\alpha$ was quantified in cell culture supernatant after $72 \mathrm{~h}$ (end of the culture) by ELISA according to manufacturer instructions (Abcam, \#ab46070). 


\section{Blood samples collection and LC-MS/MS analysis}

Blood samples were collected from 160 neurologically healthy and fasting subject (80 women/80 men) from 50 to 90 years old in EDTA tubes. Samples were centrifuged for $10 \mathrm{~min}$ (4000 rpm at $\mathrm{RT}$ ) and plasma was aliquoted and stored at $-80^{\circ} \mathrm{C}$ until analysis. Queuine being an endogenous compound in plasma, surrogate blank matrix (PBS +2\% BSA) was used for preparation of calibration standards. $10 \mu \mathrm{L}$ of a stable labeled internal standard [heavy queuine labelled with $3{ }^{15} \mathrm{~N}$ (Synthenova SAS)] was added at $0.2 \mu \mathrm{g} / \mathrm{mL}$ to $50 \mu \mathrm{L}$ of plasma before protein precipitation with acetonitrile $(\mathrm{MeCN})(150 \mu \mathrm{L})$ using a phospholipid removal plate. Samples were then centrifuged for $5 \mathrm{~min}$ at $14000 \mathrm{rpm}$ and $4^{\circ} \mathrm{C} .150 \mu \mathrm{L}$ of the supernatant was then transferred on PLR 96WP (Phree 30mg/well part No 8E-S133-TGB Phenomenex) and centrifuge at $3500 \mathrm{rpm}$ for $5 \mathrm{~min}$ at $4^{\circ} \mathrm{C}$ and then injected in LC-MS/MS. The mass spectrometer was operated in positive ionization. The chromatographic selectivity was carried out by gradient elution using $\mathrm{MeCN}$ and $10 \mathrm{mM}$ NH4OAc as mobile phases on a Hilic analytical column (ZIC-HILIC sequant $150 * 2.2 \mathrm{~mm}, 5 \mu \mathrm{m}$ column, SIL-20AC (Shimadzu) automatic sampler maintained at $4^{\circ} \mathrm{C}$, LC-20AD pump (Shimadzu)). Ionization and detection of queuine and its internal standard was performed on a triple quadrupole mass spectrometer Sciex API 5500, operating in the multiple reaction monitoring and positive ionization mode. Quantitation was done to monitor protonated precursor > product ion transition of $\mathrm{m} / \mathrm{z} 278.1>162.2$ for queuine and $281.2>166.2$ for its internal standard.

\section{Chromatographic conditions:}

Analytical column : Hilic mode Injection volume: $2 \mu \mathrm{L}$ Mobile phase A : $10 \mathrm{mM} \mathrm{NH} 4 \mathrm{OAc}$ Mobile phase B: MeCN

\begin{tabular}{|c|c|c|c|}
\hline Time (min) & $\begin{array}{c}\text { Flow rate } \\
\text { (mL/min) }\end{array}$ & \% Phase A & \% Phase B \\
\hline 0 & 0.2 & 60 & 40 \\
\hline 2 & 0.2 & 90 & 10 \\
\hline 3 & 0.2 & 90 & 10 \\
\hline 3.1 & 0.2 & 60 & 40 \\
\hline 7 & 0.2 & 60 & 40 \\
\hline & & & 55 \\
\hline
\end{tabular}

Mass spectrometry conditions: MS detector Ionisation and Acquisition mode

API 5500 (AB Sciex)

ESI (+), MRM 


\begin{tabular}{|l|l|l|l|l|l|l|}
\hline Analyte & $\begin{array}{l}\text { Parent } \\
(\mathrm{m} / \mathrm{z})\end{array}$ & $\begin{array}{l}\text { Daughter } \\
(\mathrm{m} / \mathrm{z})\end{array}$ & $\begin{array}{l}\text { Dwell } \\
(\mathrm{msec})\end{array}$ & $\begin{array}{l}\text { DP } \\
(\mathrm{V})\end{array}$ & $\begin{array}{l}\text { CE } \\
(\mathrm{eV})\end{array}$ & $\begin{array}{l}\text { CXP } \\
(\mathrm{V})\end{array}$ \\
\hline Queuine & 278.1 & 163.23 & 150 & 100 & 21 & 15 \\
\hline Internal standard & 281.2 & 166.2 & 100 & 100 & 21 & 13 \\
\hline
\end{tabular}

\section{Statistical analysis}

Data are expressed as the mean and standard error (SEM) as indicated in the figure legends. Methods used to calculate significant differences between groups are indicated in the figure's legend. Asterisks indicate statistically significant differences. A $p$-value $<0.05$ was considered statistically significant.

\section{Acknowledgments}

We would like to thank the Stellate Therapeutics team for their work, support and critical advice and discussions. We thank Eurofins for performing the LC-MS/MS analysis of queuine.

\section{Conflict of Interests}

The authors declare that they have no conflict of interest.

\section{References}

1. Stocchi F, Torti M. Constipation in Parkinson's Disease. Int Rev Neurobiol. 2017;134: 811826. doi:10.1016/bs.irn.2017.06.003

2. Beach TG, Adler CH, Sue LI, Vedders L, Lue L, White lii CL, et al. Multi-organ distribution of phosphorylated alpha-synuclein histopathology in subjects with Lewy body disorders. Acta Neuropathol. 2010;119: 689-702. doi:10.1007/s00401-010-0664-3

3. Scheperjans F, Aho V, Pereira PAB, Koskinen K, Paulin L, Pekkonen E, et al. Gut microbiota are related to Parkinson's disease and clinical phenotype. Movement Disorders. 2015;30: 350358. doi:10.1002/mds.26069

4. Boertien JM, Pereira PAB, Aho VTE, Scheperjans F. Increasing Comparability and Utility of Gut Microbiome Studies in Parkinson's Disease: A Systematic Review. J Parkinsons Dis. 2019;9: S297-S312. doi:10.3233/JPD-191711 
5. Cattaneo A, Cattane N, Galluzzi S, Provasi S, Lopizzo N, Festari C, et al. Association of brain amyloidosis with pro-inflammatory gut bacterial taxa and peripheral inflammation markers in cognitively impaired elderly. Neurobiol Aging. 2017;49: 60-68. doi:10.1016/j.neurobiolaging.2016.08.019

6. Vogt NM, Kerby RL, Dill-McFarland KA, Harding SJ, Merluzzi AP, Johnson SC, et al. Gut microbiome alterations in Alzheimer's disease. Sci Rep. 2017;7: 13537. doi:10.1038/s41598-017-13601-y

7. Zhuang Z-Q, Shen L-L, Li W-W, Fu X, Zeng F, Gui L, et al. Gut Microbiota is Altered in Patients with Alzheimer's Disease. J Alzheimers Dis. 2018;63: 1337-1346. doi:10.3233/JAD-180176

8. Sampson TR, Debelius JW, Thron T, Janssen S, Shastri GG, Ilhan ZE, et al. Gut Microbiota Regulate Motor Deficits and Neuroinflammation in a Model of Parkinson's Disease. Cell. 2016;167: 1469-1480.e12. doi:10.1016/j.cell.2016.11.018

9. Kim M-S, Kim Y, Choi H, Kim W, Park S, Lee D, et al. Transfer of a healthy microbiota reduces amyloid and tau pathology in an Alzheimer's disease animal model. Gut. 2020;69: 283294. doi:10.1136/gutjnl-2018-317431

10. Track NS. The gastrointestinal endocrine system. Can Med Assoc J. 1980;122: 287-292.

11. Fergus C, Barnes D, Alqasem M, Kelly V. The Queuine Micronutrient: Charting a Course from Microbe to Man. Nutrients. 2015;7: 2897-2929. doi:10.3390/nu7042897

12. Siard TJ, Katze JR, Farkas WR. Queuine is incorporated into brain transfer RNA. Neurochem Res. 1989;14: 1159-1164.

13. Boland C, Hayes P, Santa-Maria I, Nishimura S, Kelly VP. Queuosine formation in eukaryotic tRNA occurs via a mitochondria-localized heteromeric transglycosylase. J Biol Chem. 2009;284: 18218-18227. doi:10.1074/jbc.M109.002477

14. Suzuki T, Yashiro Y, Kikuchi I, Ishigami Y, Saito H, Matsuzawa I, et al. Complete chemical structures of human mitochondrial tRNAs. Nat Commun. 2020;11: 4269. doi:10.1038/s41467-020-18068-6

15. Nishimura S. Structure, biosynthesis, and function of queuosine in transfer RNA. Prog Nucleic Acid Res Mol Biol. 1983;28: 49-73.

16. Yokoyama S, Miyazawa T, litaka Y, Yamaizumi Z, Kasai H, Nishimura S. Three-dimensional structure of hyper-modified nucleoside $Q$ located in the wobbling position of tRNA. Nature. 1979;282: 107-109. doi:10.1038/282107a0

17. Farkas WR, Jacobson KB, Katze JR. Substrate and inhibitor specificity of tRNA-guanine ribosyltransferase. Biochim Biophys Acta. 1984;781: 64-75. doi:10.1016/01674781(84)90124-6 
18. Chen Y-C, Kelly VP, Stachura SV, Garcia GA. Characterization of the human tRNA-guanine transglycosylase: confirmation of the heterodimeric subunit structure. RNA. 2010;16: 958968. doi:10.1261/rna.1997610

19. Grosjean H, de Crécy-Lagard V, Marck C. Deciphering synonymous codons in the three domains of life: co-evolution with specific tRNA modification enzymes. FEBS Lett. 2010;584: 252-264. doi:10.1016/j.febslet.2009.11.052

20. Müller M, Legrand C, Tuorto F, Kelly VP, Atlasi Y, Lyko F, et al. Queuine links translational control in eukaryotes to a micronutrient from bacteria. Nucleic Acids Res. 2019;47: 37113727. doi:10.1093/nar/gkz063

21. Tuorto F, Legrand C, Cirzi C, Federico G, Liebers R, Müller M, et al. Queuosine-modified tRNAs confer nutritional control of protein translation. EMBO J. 2018;37.

doi:10.15252/embj.201899777

22. Zaborske JM, DuMont VLB, Wallace EWJ, Pan T, Aquadro CF, Drummond DA. A nutrientdriven tRNA modification alters translational fidelity and genome-wide protein coding across an animal genus. PLoS Biol. 2014;12: e1002015. doi:10.1371/journal.pbio.1002015

23. Anderson S, Bankier AT, Barrell BG, de Bruijn MH, Coulson AR, Drouin J, et al. Sequence and organization of the human mitochondrial genome. Nature. 1981;290: 457-465. doi:10.1038/290457a0

24. Cabral-Costa JV, Kowaltowski AJ. Neurological disorders and mitochondria. Mol Aspects Med. 2020;71: 100826. doi:10.1016/j.mam.2019.10.003

25. Skolnick SD, Greig NH. Microbes and Monoamines: Potential Neuropsychiatric Consequences of Dysbiosis. Trends in Neurosciences. 2019;42: 151-163. doi:10.1016/j.tins.2018.12.005

26. Marks T, Farkas WR. Effects of a diet deficient in tyrosine and queuine on germfree mice. Biochem Biophys Res Commun. 1997;230: 233-237. doi:10.1006/bbrc.1996.5768

27. Rakovich T, Boland C, Bernstein I, Chikwana VM, Iwata-Reuyl D, Kelly VP. Queuosine Deficiency in Eukaryotes Compromises Tyrosine Production through Increased Tetrahydrobiopterin Oxidation. J Biol Chem. 2011;286: 19354-19363. doi:10.1074/jbc.M111.219576

28. Medeiros CAM, Carvalhedo de Bruin PF, Lopes LA, Magalhães MC, de Lourdes Seabra M, Sales de Bruin VM. Effect of exogenous melatonin on sleep and motor dysfunction in Parkinson's disease. J Neurol. 2007;254: 459-464. doi:10.1007/s00415-006-0390-x

29. Reisser $\mathrm{T}$, Langgut $\mathrm{W}$, Kersten $\mathrm{H}$. The nutrient factor queuine protects HeLa cells from hypoxic stress and improves metabolic adaptation to oxygen availability. Eur J Biochem. 1994;221: 979-986. doi:10.1111/j.1432-1033.1994.tb18814.x 
30. Langgut W, Reisser T, Kersten H. Queuine modulates growth of HeLa cells depending on oxygen availability. Biofactors. 1990;2: 245-249.

31. Brooks A, Garcia G, Showalter H. A short, concise synthesis of queuine. Tetrahedron Letters - TETRAHEDRON LETT. 2010;51: 4163-4165. doi:10.1016/j.tetlet.2010.06.008

32. Volpicelli-Daley LA, Luk KC, Patel TP, Tanik SA, Riddle DM, Stieber A, et al. Exogenous $\alpha$ Synuclein Fibrils Induce Lewy Body Pathology Leading to Synaptic Dysfunction and Neuron Death. Neuron. 2011;72: 57-71. doi:10.1016/j.neuron.2011.08.033

33. Fernagut P-O, Chalon S, Diguet E, Guilloteau D, Tison F, Jaber M. Motor behaviour deficits and their histopathological and functional correlates in the nigrostriatal system of dopamine transporter knockout mice. Neuroscience. 2003;116: 1123-1130. doi:10.1016/s0306-4522(02)00778-9

34. Dauer W, Przedborski S. Parkinson's disease: mechanisms and models. Neuron. 2003;39: 889-909. doi:10.1016/s0896-6273(03)00568-3

35. Sakono M, Zako T. Amyloid oligomers: formation and toxicity of Abeta oligomers. FEBS J. 2010;277: 1348-1358. doi:10.1111/j.1742-4658.2010.07568.x

36. Callizot N, Combes M, Steinschneider R, Poindron P. Operational dissection of $\beta$-amyloid cytopathic effects on cultured neurons. Journal of Neuroscience Research. 2013;91: 706716. doi:10.1002/jnr.23193

37. Jan A, Hartley DM, Lashuel HA. Preparation and characterization of toxic Abeta aggregates for structural and functional studies in Alzheimer's disease research. Nat Protoc. 2010;5: 1186-1209. doi:10.1038/nprot.2010.72

38. Pearse RV, Young-Pearse TL. Lost in translational biology: Understanding sex differences to inform studies of diseases of the nervous system. Brain Res. 2019;1722: 146352. doi:10.1016/j.brainres.2019.146352

39. Duechler M, Leszczyńska G, Sochacka E, Nawrot B. Nucleoside modifications in the regulation of gene expression: focus on tRNA. Cell Mol Life Sci. 2016;73: 3075-3095. doi:10.1007/s00018-016-2217-y

40. Boccaletto P, Machnicka MA, Purta E, Piatkowski P, Baginski B, Wirecki TK, et al. MODOMICS: a database of RNA modification pathways. 2017 update. Nucleic Acids Res. 2018;46: D303-D307. doi:10.1093/nar/gkx1030

41. Bednářová $A$, Hanna $M$, Durham I, VanCleave $T$, England $A$, Chaudhuri $A$, et al. Lost in Translation: Defects in Transfer RNA Modifications and Neurological Disorders. Frontiers in Molecular Neuroscience. 2017;10. doi:10.3389/fnmol.2017.00135 
42. Fu H, Feng J, Liu Q, Sun F, Tie Y, Zhu J, et al. Stress induces tRNA cleavage by angiogenin in mammalian cells. FEBS Lett. 2009;583: 437-442. doi:10.1016/j.febslet.2008.12.043

43. Wang X, Matuszek Z, Huang Y, Parisien M, Dai Q, Clark W, et al. Queuosine modification protects cognate tRNAs against ribonuclease cleavage. RNA. 2018;24: 1305-1313. doi:10.1261/rna.067033.118

44. Li S, Xu Z, Sheng J. tRNA-Derived Small RNA: A Novel Regulatory Small Non-Coding RNA. Genes (Basel). 2018;9. doi:10.3390/genes9050246

45. Li S, Hu G-F. Emerging role of angiogenin in stress response and cell survival under adverse conditions. J Cell Physiol. 2012;227: 2822-2826. doi:10.1002/jcp.23051

46. Yamasaki S, Ivanov P, Hu G-F, Anderson P. Angiogenin cleaves tRNA and promotes stressinduced translational repression. J Cell Biol. 2009;185: 35-42. doi:10.1083/jcb.200811106

47. Ivanov P, Emara MM, Villen J, Gygi SP, Anderson P. Angiogenin-Induced tRNA Fragments Inhibit Translation Initiation. Molecular Cell. 2011;43: 613-623.

doi:10.1016/j.molcel.2011.06.022

48. Magee R, Londin E, Rigoutsos I. TRNA-derived fragments as sex-dependent circulating candidate biomarkers for Parkinson's disease. Parkinsonism Relat Disord. 2019. doi:10.1016/j.parkreldis.2019.05.035

49. Müller M, Hartmann M, Schuster I, Bender S, Thüring KL, Helm M, et al. Dynamic modulation of Dnmt2-dependent tRNA methylation by the micronutrient queuine. Nucleic Acids Res. 2015;43: 10952-10962. doi:10.1093/nar/gkv980

50. Schaefer M, Pollex T, Hanna K, Tuorto F, Meusburger M, Helm M, et al. RNA methylation by Dnmt2 protects transfer RNAs against stress-induced cleavage. Genes Dev. 2010;24: 1590-1595. doi:10.1101/gad.586710

51. Crivello M, O'Riordan SL, Woods I, Cannon S, Halang L, Coughlan KS, et al. Pleiotropic activity of systemically delivered angiogenin in the SOD1G93A mouse model. Neuropharmacology. 2018;133: 503-511. doi:10.1016/j.neuropharm.2018.02.022

52. Subramanian $\mathrm{V}$, Feng $\mathrm{Y}$. A new role for angiogenin in neurite growth and pathfinding: implications for amyotrophic lateral sclerosis. Hum Mol Genet. 2007;16: 1445-1453. doi:10.1093/hmg/ddm095

53. Kieran D, Sebastia J, Greenway MJ, King MA, Connaughton D, Concannon CG, et al. Control of motoneuron survival by angiogenin. J Neurosci. 2008;28: 14056-14061. doi:10.1523/JNEUROSCI.3399-08.2008 
54. van Es MA, Schelhaas HJ, van Vught PWJ, Ticozzi N, Andersen PM, Groen EJN, et al. Angiogenin variants in Parkinson disease and amyotrophic lateral sclerosis. Ann Neurol. 2011;70: 964-973. doi:10.1002/ana.22611

55. Steidinger TU, Slone SR, Ding H, Standaert DG, Yacoubian TA. Angiogenin in Parkinson disease models: role of Akt phosphorylation and evaluation of AAV-mediated angiogenin expression in MPTP treated mice. PLoS ONE. 2013;8: e56092. doi:10.1371/journal.pone.0056092

56. Ichinose $\mathrm{H}$, Inoue $\mathrm{K}-\mathrm{I}$, Arakawa $\mathrm{S}$, Watanabe $\mathrm{Y}$, Kurosaki $\mathrm{H}$, Koshiba $\mathrm{S}$, et al. Alterations in the reduced pteridine contents in the cerebrospinal fluids of LRRK2 mutation carriers and patients with Parkinson's disease. J Neural Transm (Vienna). 2018;125: 45-52. doi:10.1007/s00702-017-1784-x

57. Chen C, Turnbull DM, Reeve AK. Mitochondrial Dysfunction in Parkinson's Disease-Cause or Consequence? Biology (Basel). 2019;8. doi:10.3390/biology8020038

58. Swerdlow RH. Mitochondria and Mitochondrial Cascades in Alzheimer's Disease. Journal of Alzheimer's disease: JAD. 2018;62: 1403-1416. doi:10.3233/JAD-170585

59. Suzuki T, Nagao A, Suzuki T. Human mitochondrial tRNAs: biogenesis, function, structural aspects, and diseases. Annu Rev Genet. 2011;45: 299-329. doi:10.1146/annurev-genet110410-132531

60. Braymer JJ, Lill R. Iron-sulfur cluster biogenesis and trafficking in mitochondria. J Biol Chem. 2017;292: 12754-12763. doi:10.1074/jbc.R117.787101

61. Sorrentino V, Romani M, Mouchiroud L, Beck JS, Zhang H, D'Amico D, et al. Enhancing mitochondrial proteostasis reduces amyloid- $\beta$ proteotoxicity. Nature. 2017;552: 187-193. doi:10.1038/nature25143

62. Hayes P, Fergus C, Ghanim M, Cirzi C, Burtnyak L, McGrenaghan CJ, et al. Queuine Micronutrient Deficiency Promotes Warburg Metabolism and Reversal of the Mitochondrial ATP Synthase in Hela Cells. Nutrients. 2020;12. doi:10.3390/nu12030871

63. Dirheimer G, Baranowski W, Keith G. Variations in tRNA modifications, particularly of their queuine content in higher eukaryotes. Its relation to malignancy grading. Biochimie. 1995;77: 99-103. doi:10.1016/0300-9084(96)88111-9

64. Varghese $\mathrm{S}$, Cotter $\mathrm{M}$, Chevot F, Fergus $\mathrm{C}$, Cunningham $\mathrm{C}$, Mills $\mathrm{KH}$, et al. In vivo modification of tRNA with an artificial nucleobase leads to full disease remission in an animal model of multiple sclerosis. Nucleic Acids Res. 2016; gkw847. doi:10.1093/nar/gkw847 


\section{Supporting information}

S1 Fig. STL-101 protects against chronic injury of rat cortical neurons with $A \beta_{1-42 .}(A)$ Quantification of neurite network after $A \beta_{1-42}(5 \mu \mathrm{M})$ injury and STL-101 pre-treatment at the indicated concentrations. (B) $p$-tau quantification in neurites after $A \beta$ 1-42 injury. (C) Neuron survival after $A \beta_{1 \text { - }}$ 42 injury and STL-101 pre-treatment. (D) Measurement of TNF- $\alpha$ release after $A \beta_{1-42}$ injury and STL-101 pre-treatment. Results are expressed as a percentage of control condition and mean +/$\operatorname{SEM}(\mathrm{n}=4-6)$ is shown. ${ }^{*} \mathrm{p}<0.05,{ }^{*} \mathrm{p}<0.01, * * * \mathrm{p}<0.001, * * * * \mathrm{p}<0.0001$ (One-way ANOVA followed by Dunnett's multiple comparison test).

S2 Fig. The level of queuine is lower in female mice than male. Measurement of queuine level was performed by LC-MS/MS in plasma of 6 female and 8 male mice of 45 weeks of age. $* * * * p<0.0001$ (unpaired t-test).

\section{Figures legend}

Fig 1. STL-101 decreases $\alpha$-syn pSer129 in an in vitro model of synucleinopathy and protects dopaminergic (DA) neurons from MPP+ injury. (A) IF of human $\alpha$-syn (labelled in red with syn211 antibody) and the phosphorylated/aggregated form of $\alpha$-syn ( $\alpha$-syn pSer129, labelled in green with EP1536Y antibody) on control and huPFFs-treated mouse cortical neurons. Scale bar = $200 \mu \mathrm{m}$. (B) IF of $\alpha$-syn pSer129 (EP1536Y antibody) three weeks after huPFFs exposure in untreated (-) and treated mouse cortical neurons with STL-101 at 100nM. Scale bar $=200 \mu \mathrm{m}$. (C) Quantification of $\alpha$-syn pSer129 as shown in B. in control (no huPFFs) and huPFFs-exposed mouse cortical neurons

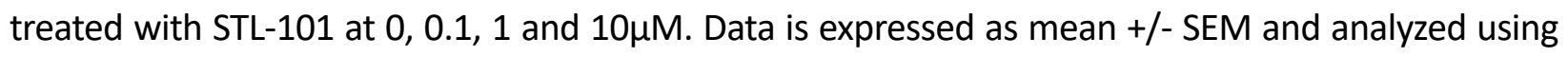
two-way ANOVA followed by Dunnett's multiple comparison; ***p<0.001 cf. huPFFs $+[\mathrm{STL}-101]=$ O $\mu \mathrm{M}$. (D) STL-101 was added at the indicated concentrations to rat DA neuron cultures 1 day

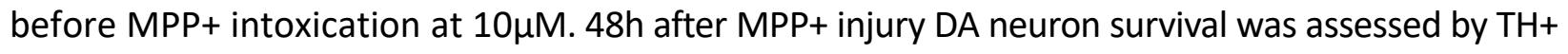
neurons counting. Statistical significance was calculated using one-way ANOVA, Dunnett's multiple 
comparison test $(* p<0.05, * * * * p<0.0001) . \mathrm{n}=3$. Dimethyl fumarate $(\mathrm{DMF})$ was used at $10 \mu \mathrm{M}$ as a positive control.

Fig 2. STL-101 protects against acute injury with $A \boldsymbol{\beta}_{1-42}$ in rat cortical neurons. $(A)$ Representative IF of MAP2 (red) and p-tau (AT100, green) in control (-), $A \beta_{1-42}$ injured neurons, $A \beta_{1-42}$ injured neurons pre-treated with STL-101 at 300nM for $24 \mathrm{~h}$ and $A \beta_{1-42}$ injured neurons treated with BDNF at 50ng/mL. A $\beta_{1-42}$ was used at $20 \mu \mathrm{M}$. Scale bar $=100 \mu \mathrm{m}$. (B) Quantification of $p$-tau in MAP2/AT100 IFs as shown in A. and STL-101 pre-treatment at the indicated concentrations. (C) Measurement of neurite network as shown in A. (D) Neuron survival quantification after $A \beta_{1-42}$ injury. STL-101 pretreatments were performed at the indicated concentrations and the neurotrophic factor BDNF was used as a positive control. Results are expressed as a percentage of control condition and mean +/- SEM ( $n=4-6)$ is shown. Statistical analysis was performed using one-way ANOVA followed by Dunnett's multiple comparison test $\left({ }^{*} p<0.05,{ }^{* *} p<0.01, * * * p<0.001, * * * * p<0.0001\right)$.

Fig 3. Queuine does not vary with age but is higher in women than men. (A) Measurement of queuine level was performed by LC-MS/MS in plasma samples of 80 neurologically healthy women and 80 neurologically healthy men from 50 to 90 years old. (B) Comparison of queuine level between men and women as shown in $A .{ }^{* *} p<0.01$ (Mann Whitney test). 
A
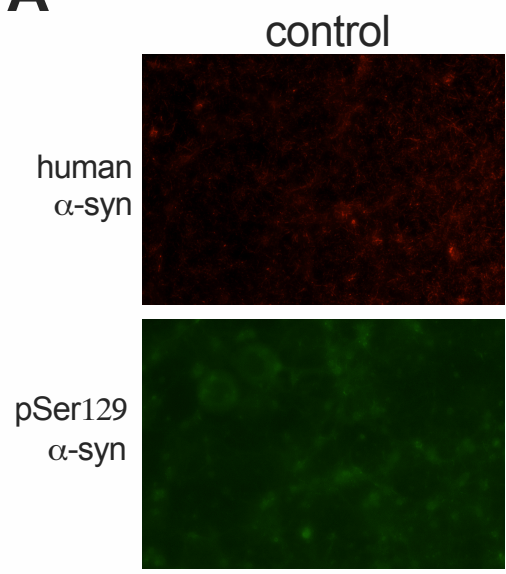

phase contrast
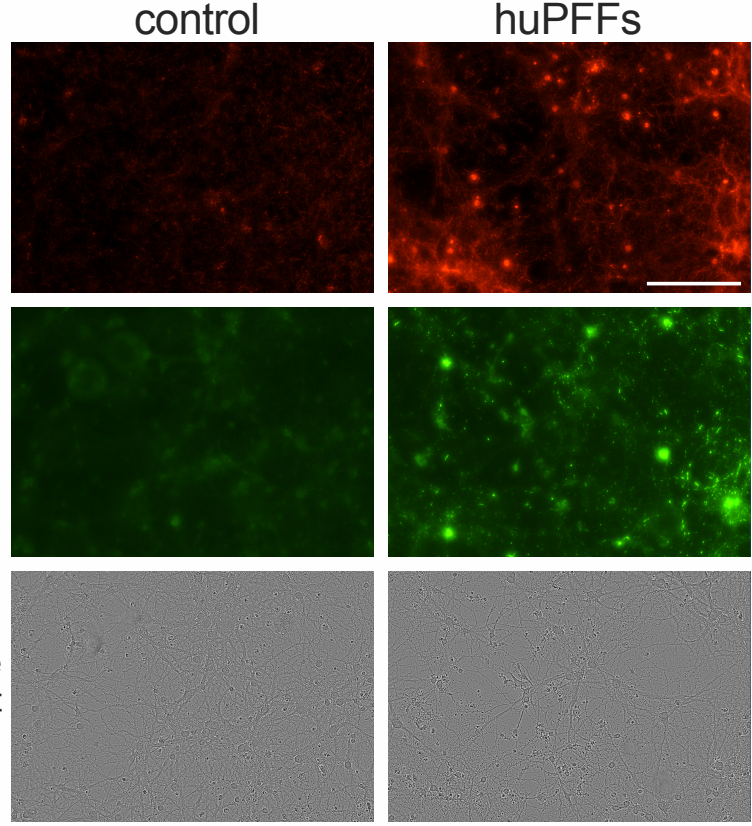

D

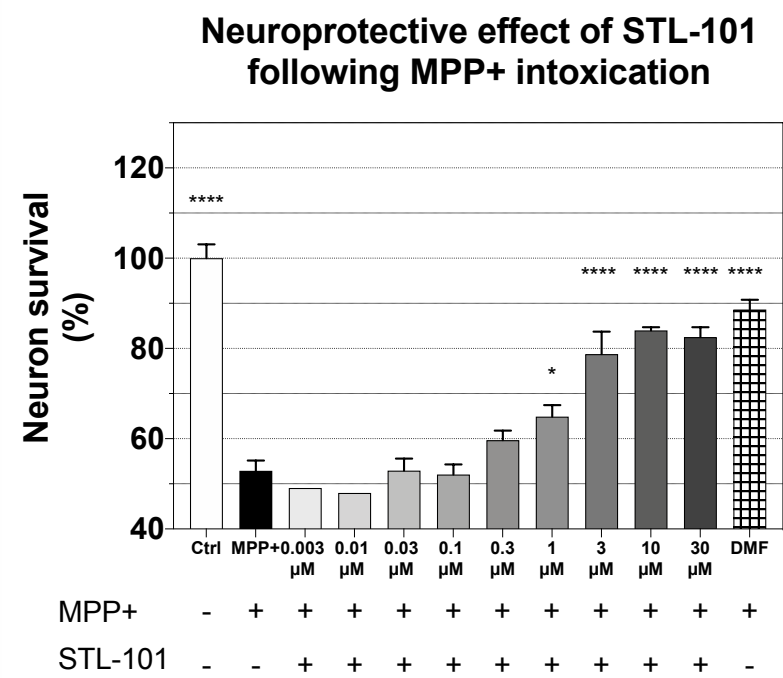

B

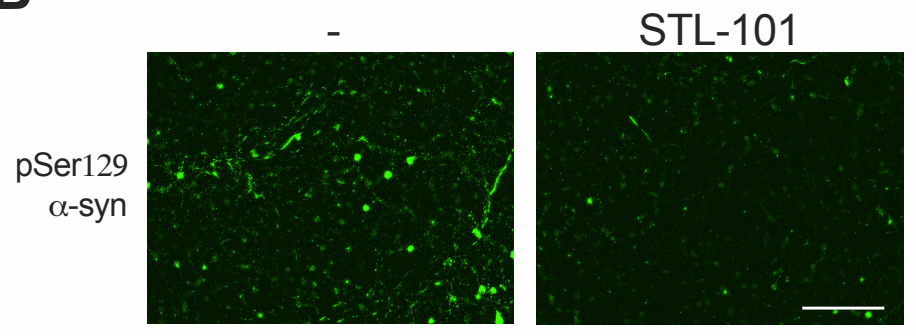

C

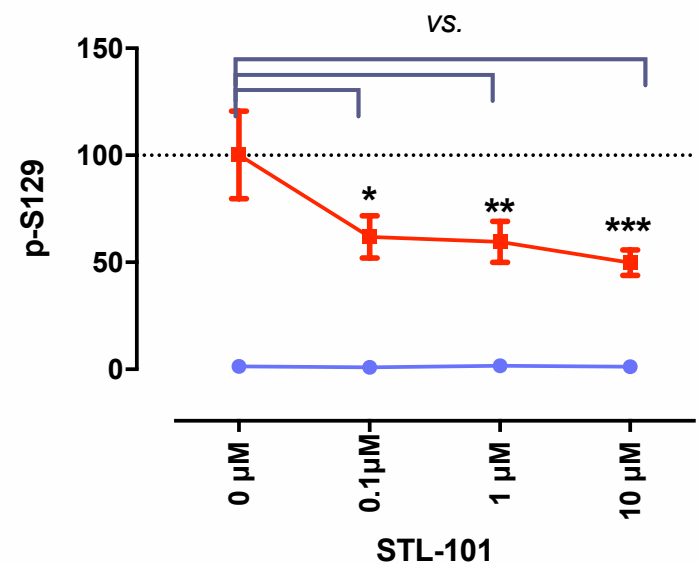

- control

$\rightarrow$ huPFFs

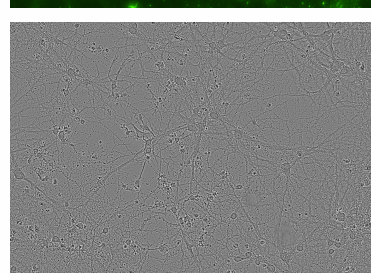

STL-101 


\section{A}
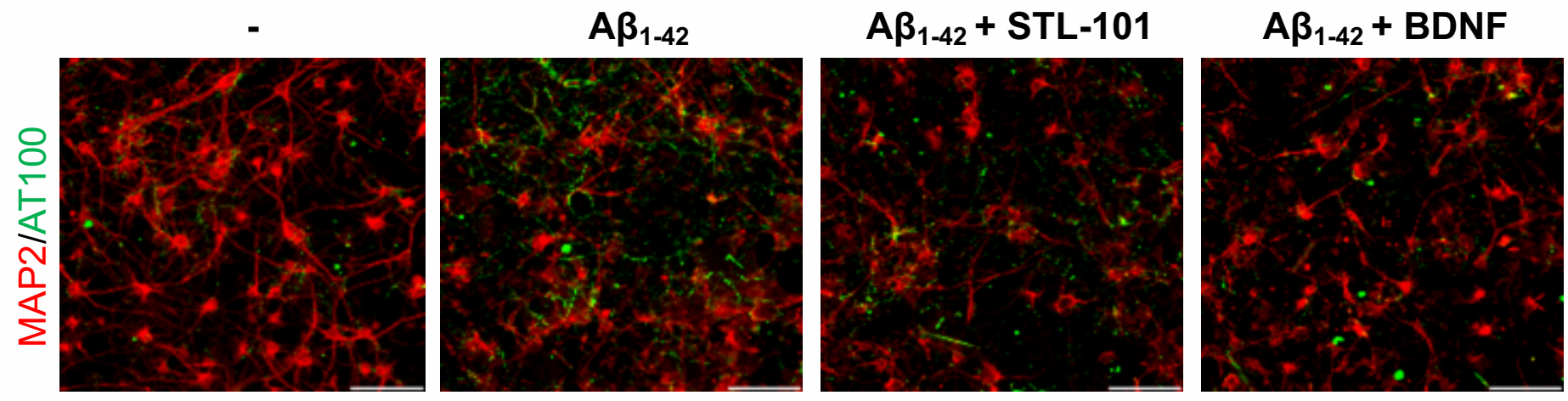

B
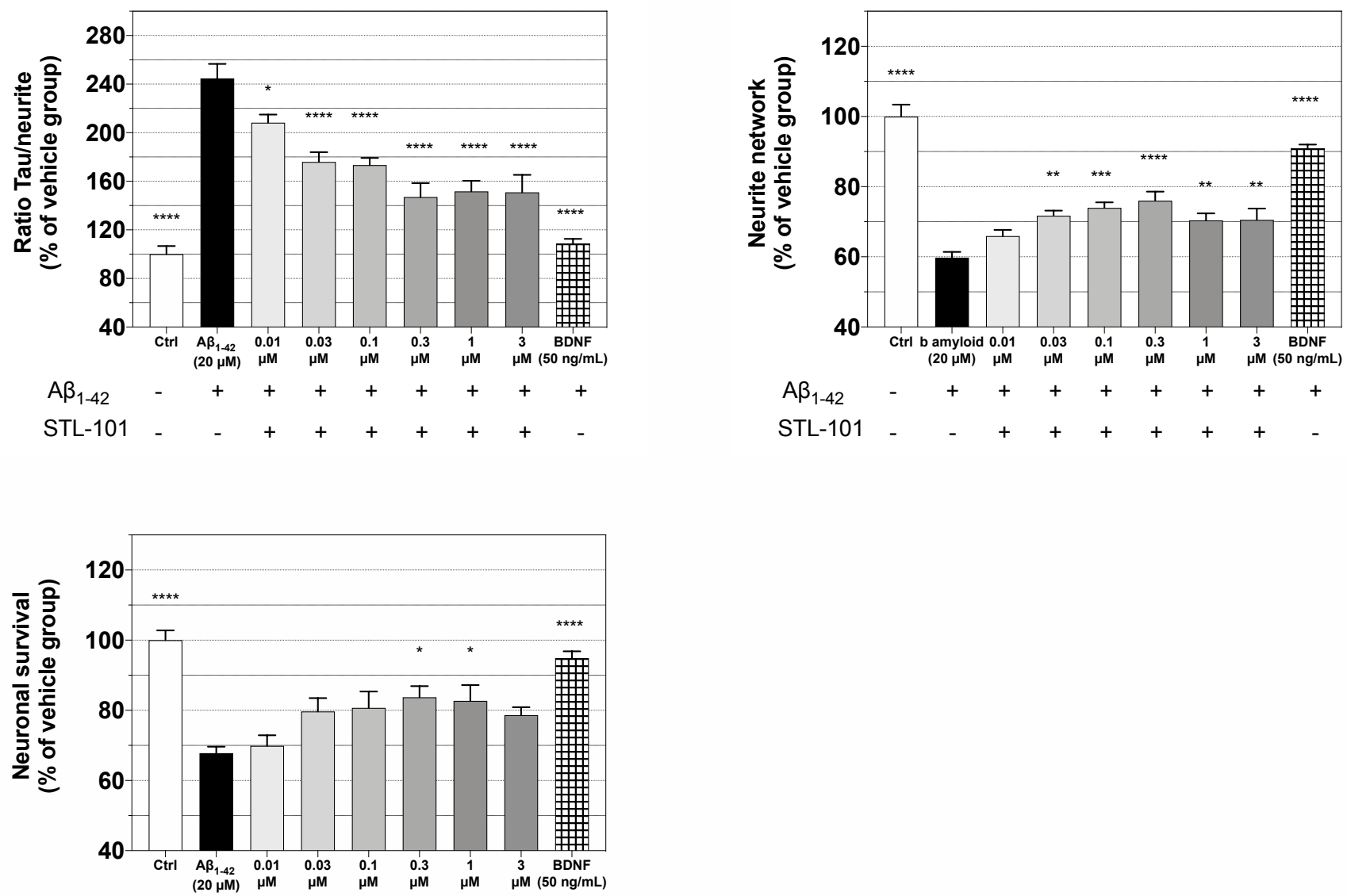

$A \beta_{1-42}$

STL-101 
Figure 3

A

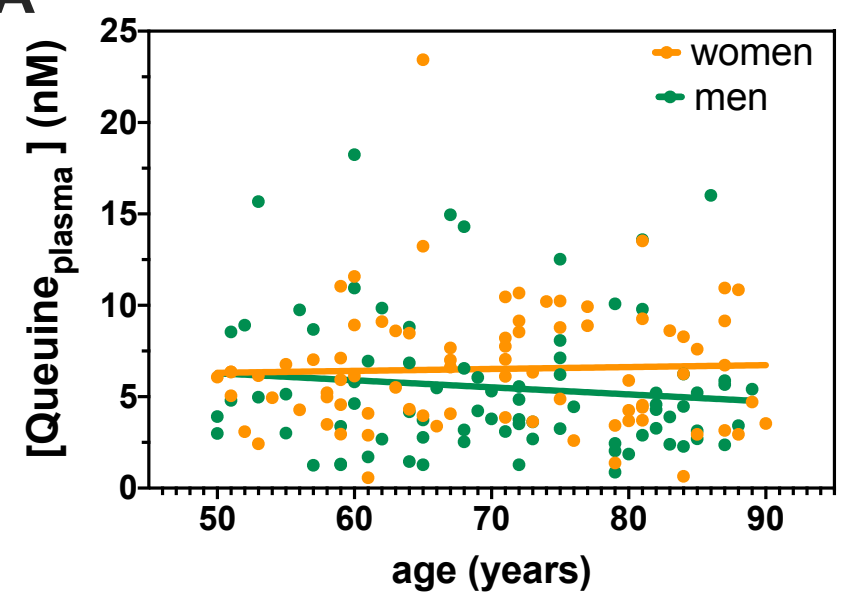

B

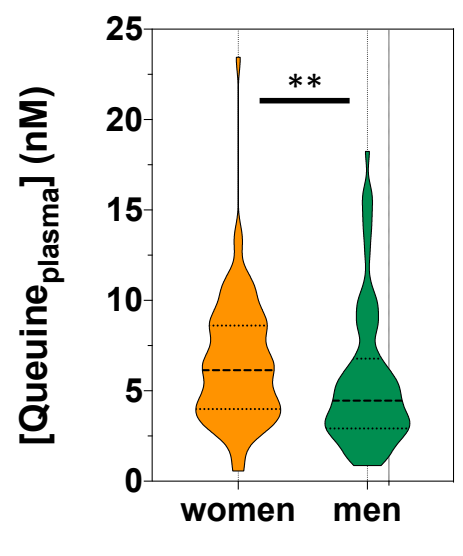


bioRxiv preprint doi: https://doi.org/10.1101/2021.01.20.427538; this version posted January 22, 2021. The copyright holder for this preprint (which was not certified by peer review) is the author/funder. All rights reserved. No reuse allowed without permission.

Figure S1

A

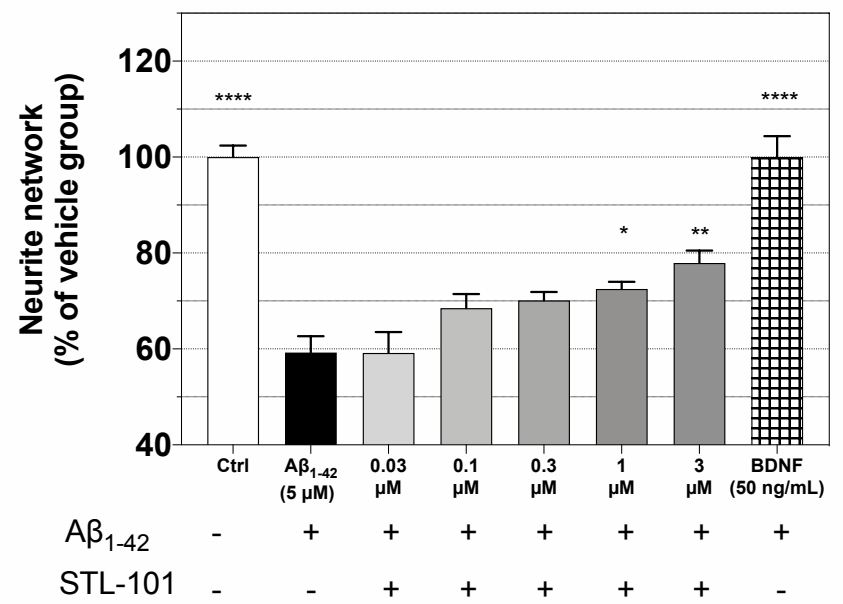

C

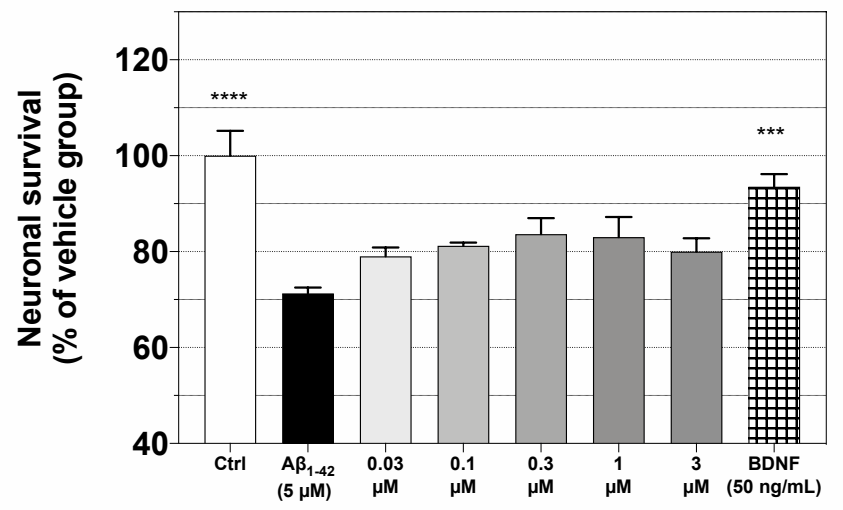

B

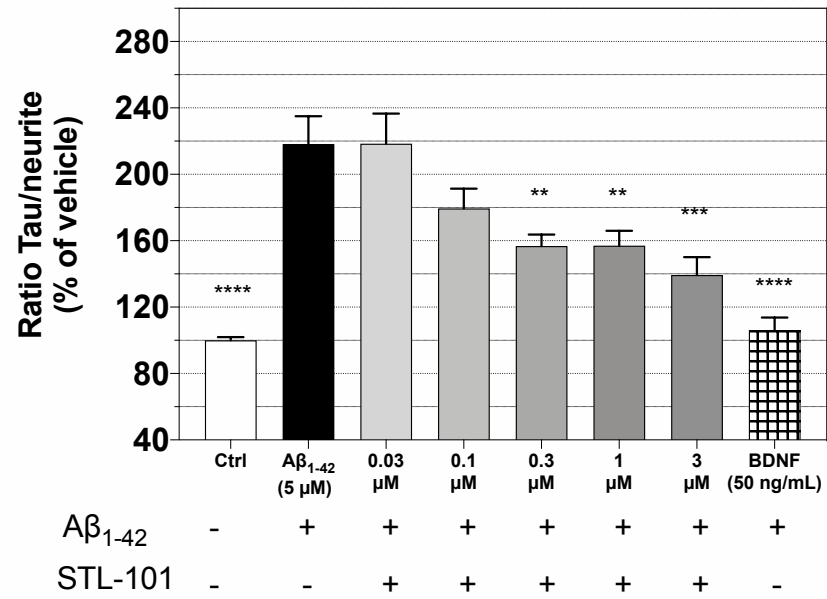

D

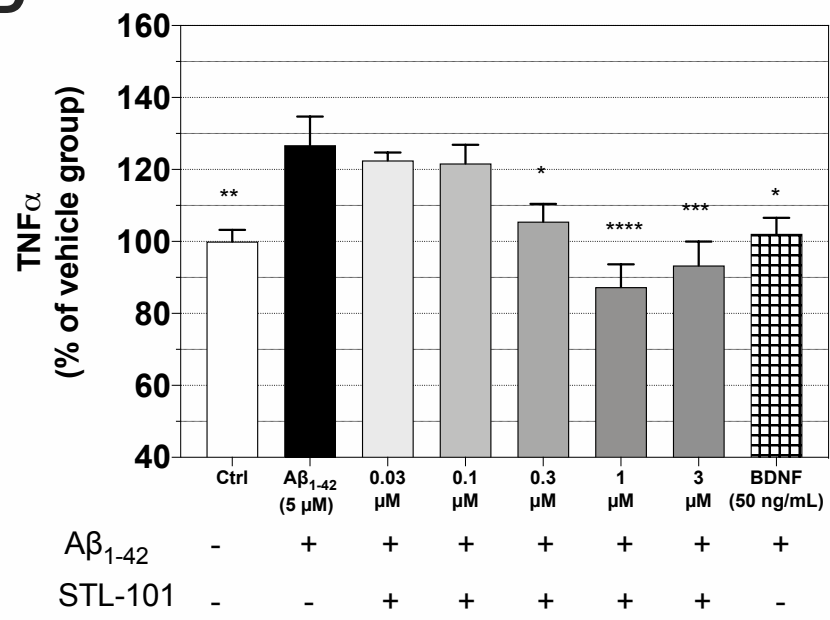


bioRxiv preprint doi: https://doi.org/10.1101/2021.01.20.427538; this version posted January 22, 2021. The copyright holder for this preprint (which was not certified by peer review) is the author/funder. All rights reserved. No reuse allowed without permission.

Figure S2

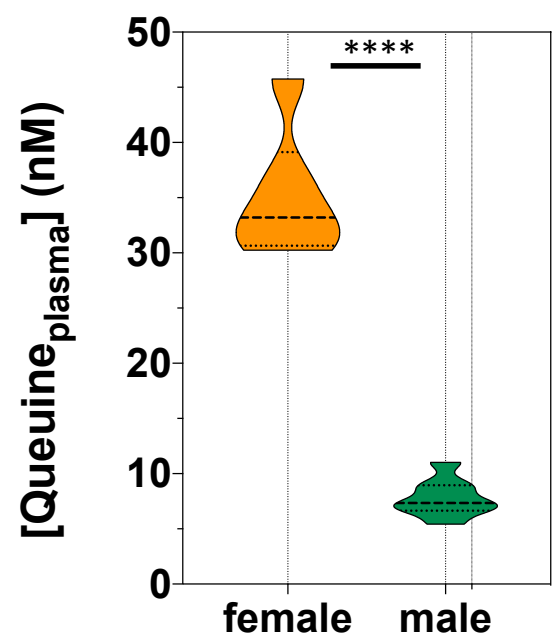

